\title{
Coming full circle: From antibiotics to probiotics and prebiotics
}

\author{
John M Conly MD¹, B Lynn Johnston MD²
}

A ntibiotics are used as agents to prevent and treat infections caused by pathogenic bacteria and other microbes, and rank as one of the most important developments of modern medicine. The word 'antibiotic' is derived from the Greek term 'biotikos' and may be literally translated as 'against life'. Antibiotics were first used centuries ago by the Chinese in the form mouldy soybean curd applied to carbuncles and furuncles. (1) The ancient Greeks, including Hippocrates, routinely used agents with antimicrobial properties such as myrrh and inorganic salts in their treatment of infected wounds (1). The discovery of penicillin by Fleming in 1928, followed by the discovery and clinical use of sulphonamides in the 1930s, heralded the age of modern antibiotherapy $(1,2)$. Penicillin usage became widespread in the 1940s during the war years and by the 1950s the 'golden era' of antibiotic development and use was well underway.

Probiotics are live microbes that are used as agents to alter the composition or metabolic activities of the microbiota, or to modulate immune system reactivity in a way that benefits health $(3,4)$. The word 'probiotic' is also derived from the same Greek term 'biotikos' which may be literally translated as 'for life'. Probiotics have been used for many years in the animal feed industry, but they are now being increasingly made available in many forms and can be purchased over the counter as freeze-dried preparations in health food stores. Prebiotics are food ingredients, usually oligosaccharides, that escape digestion in the upper gastrointestinal tract and selectively stimulate the growth of selective bacterial genera such as bifidobacteria and lactobacilli in the colon $(4,5)$. It is believed that modulation of the normal microflora to benefit the host can be achieved through the use of prebiotics and probiotics. There is now increasing evidence that selected probiotic strains can provide health benefits to their human hosts and it is noteworthy that the Food and Agriculture Organization of the United Nations and the World Health Organization have stated that there is adequate scientific evidence to indicate that there is potential for probiotics to provide health benefits (6). Given these recent developments, it was considered timely to review the background and conceptual framework of the use of these agents and the evidence for their effectiveness in clinical settings.
The human intestine contains a complex, dynamic and diverse number of bacteria, that may be differentiated into native inhabitants and transient flora (7). These microorganisms colonize the mucosal surface of the oral cavity, the upper respiratory tract, much of the gastrointestinal tract and the urogenital tract. Although the gastrointestinal tract is sterile at birth, microflora colonize the mucosal surfaces of infants during an ecological succession of organisms that differ from the adult microflora (7). The composition of the flora is influenced heavily by the receipt of oral formula or breast milk. Breastfed infants have been found to have an increased number of Bifidobacteria but rarely have Clostridium species, whereas formulafed infants have large numbers of Lactobacilli, Bacteroides and Clostridium, and relatively few Bifidobacterium species. As solid foods are added to the infant's diet, the microflora becomes similar regardless of breast feeding status, with Bacteroides and anaerobic Gram-positive cocci appearing in the flora. After the infant reaches two years of age, a conversion to normal adult flora begins, and populations of Bacteroides and anaerobic cocci increase until they equal or exceed those of Bifidobacterium. The number of Gram-negative anaerobes increases to adult levels, whereas coliform, clostridial and streptococcal populations decrease to the levels found in healthy adults. The development of the microflora from the neonatal to the adult composition is very important with respect to the development of the intestinal mucosal immune system and its ability to discriminate between pathogenic microorganisms and the vast array of antigens to which it is exposed over a lifetime. The intestinal mucosa thus has the unique properties of tolerance to environmental antigens (which may include probiotics) and specific immunological responsiveness to mucosal pathogenic microorganisms. The phenomenon of tolerance is thought to occur through clonal deletion, clonal anergy of antigen-specific $\mathrm{T}$ cells, or immune deviation medicated via Class I restricted $\mathrm{CD}^{+} \mathrm{T}$ cells and cytokines such as interleukin (IL)-10, or transforming growth factor- $\beta$ (7).The process is dependent on the normal indigenous flora because germ free animals are defective with respect to tolerance. The immunological response of the gut is mediated through lymphocytes within the lamina propria and both T helper cell 1 (Th-1; IL-12, IFN- $\gamma$ ) and T helper cell-2 (IL-10, IL-4, IL-5) responses

\footnotetext{
${ }^{1}$ Departments of Pathology and Laboratory Medicine, Medicine, and Microbiology and Infectious Diseases, University of Calgary, Calgary, Alberta; ${ }^{2}$ Queen Elizabeth II Health Sciences Centre and Dalhousie University, Halifax, Nova Scotia

Correspondence: Dr John Conly, Departments of Pathology and Laboratory Medicine, Medicine, and Microbiology and Infectious Diseases, Room 930, 9th Floor, North Tower, 1403 29th Street Northwest, Calgary, Alberta T2N 2T9. Telephone 403-944-8222, fax 403-944-1095, e-mail jconly@ucalgary.ca

Received and accepted for publication May 25, 2004
} 
may be elicited directed towards intracellular and extracellular pathogens, respectively. In addition, the mucosal microflora have the capacity for inhibitory or bactericidal activity towards transient microbial pathogens within the gut in a process termed microbial interference.

The latter explains one of the postulated mechanisms whereby probiotics exert their protective or therapeutic effects. The beneficial bacteria prevent colonization of pathogenic microorganisms by competitive inhibition for microbial adhesion sites. For example, Lactobacillus casei strain GG and Lactobacillus plantarum have demonstrated the ability to competitively inhibit the attachment of enteropathogenic Escherichia coli (8) and Saccharomyces boulardii has been shown to decrease in vitro attachment of Entamoeba histolytica trophozoites to erythrocytes (9). Another postulated mechanism for the effects of probiotics is the production of organic acids, fatty free acids, ammonia, hydrogen peroxide and bacteriocins, all of which have antimicrobial activity. $L$ casei strain $G G$ produces a low-molecular-weight antibacterial substance that is inhibitory to both Gram-positive and Gram-negative enteric bacteria (10). Another mechanism of probiotic activity is the production of enzymes that modify toxin receptors or block toxin-mediated effects, exemplified by the degradation of Clostridium difficile toxin receptors in the rabbit ileum by $S$ boulardii $(11,12)$. Probiotics may also have significant contributions on intestinal mucosal immunity. Several studies have demonstrated adjuvant-like effects on intestinal and systemic immunity by oral administration of different probiotics, particularly in the stimulation of enhanced immunoglobulin A responses to pathogenic viruses (13-15) and may also enhance phagocytic activity against intracellular pathogens (16).

The use of probiotics in the form of fermented foods has been commonplace for many years and the benefits of foods containing live bacteria were recognized centuries ago. However, the historical perspective on concepts related to intestinal microecology date back to Elie Metchnikoff (7) in the early part of the 20th century, and he is considered the 'father' of probiotics. Metchinkoff proposed a scientific rationale for the beneficial effects of bacteria in yogurt and attributed the long life of Bulgarian peasants to their intake of yogurt containing Lactobacillus species (7). There are several commercially available supplements containing viable microorganisms with probiotic properties, either in lyophilized form or as fermented food products. The most commonly used probiotics are the lactic acid bacteria including various Lactobacillus, Enterococcus and Bifidobacterium species and nonpathogenic ascospore yeasts, principally $S$ boulardii.

Specific areas of potential use of probiotics that have been proposed in the past 50 years include the prevention and

\section{REFERENCES}

1. Weinstein L. General considerations. In: Goodman LS, Gilman A, eds. The Pharmacological Basis of Therapeutics. New York: Macmillan, 1970:1154.

2. Fleming A. Nobel Lecture, December 11, 1945. Nobel e-Museum. <http://www.nobel.se/medicine/laureates/1945/fleming-lecture.html> (Version current at May 25, 2004).

3. Reid G, Jass J, Sebulsky MT, McCormick JK. Potential uses of probiotics in clinical practice. Clin Micro Rev 2003;16:658-72.

4. Mcfarlane G, Cummings JH. Probiotics and prebiotics: Can regulating the activities of intestinal bacteria benefit health? BMJ 1999;318:999-1003. treatment of diarrheal diseases in adults and children, prevention of vaginitis and urinary tract infection in adults, food allergy prevention, and antitumour action in the gut, bladder and cervix. Multiple properties of probiotics have been suggested as potential protective factors in the digestive system against microorganisms such as enteropathogenic E coli, Salmonella, Listeria species and Helicobacter pylori $(3,7)$. However, it is only recently that the scientific knowledge and tools have become available to properly evaluate the effects of probiotics on normal health and well being, and their potential in preventing and treating disease. A recent review of the clinical trials in support of the beneficial effect of probiotics has been published (3). The most supportive evidence of a beneficial effect of probiotics has been established with Lactobacillus rhamnosus GG and Bifidobacterium lactis BB-12 for prevention and Lactobacillus reuteri SD2222 for the treatment of acute diarrhea caused by rotaviruses in children based on randomized, double blinded and placebo-controlled trials. A statistically significant reduction in the duration of diarrhea was reported in several of the trials. A recent metaanalysis evaluated trials on the efficacy of $S$ boulardii and lactobacilli in the prevention and treatment of diarrhea associated with the use of antibiotics and revealed an odds ratio of 0.39 (95\% CI, 0.25 to $0.62 ; \mathrm{P}<0.001)$ and 0.34 (95\% CI, 0.19 to $0.61 ; \mathrm{P}<0.01)$, respectively, in favour of active treatment over placebo (17). Additional clinical trials have suggested a reduction in recurrences of $H$ pylori, alleviation of symptoms of inflammatory bowel disease, carcinogen reduction, allergy reduction, reduction in the occurrence of recurrent urinary infection, and a reduction in recurrences of bacterial vaginosis (3) but the trials were smaller and had less power. The use of probiotics has been advocated by some as a means of reducing or eliminating colonization with antibiotic-resistant microbes (7) and this is another area that requires more study.

Although the use of probiotics may be beneficial in certain settings, one unresolved concern is whether commercially available products matched their claims from both a quantitative and qualitative perspective. Two recent studies, including a Canadian study (18) have suggested that there may be significant differences in the batches of probiotic preparations. The findings match another study that was conducted in Britain (19). These studies serve to highlight the need for better quality control on these products.

The role of probiotics and prebiotics will likely increase in the future as evidence accrues from well conducted studies on the efficacy of these agents when used in standardized and regulated formulations. The potential for probiotics to be used as an adjunct in the control of antibiotic resistance is particularly appealing.

5. Tuohy KM, Probert HM, Smejkal CW, Gibson GR. Using probiotics and prebiotics to improve gut health. Drug Discov Today 2003;8:692-700.

6. Food and Agriculture Organization of the United Nations and World Health Organization. 2001 (posting date). Regulatory and clinical aspects of dairy probiotics. Food and Agriculture

Organization of the United Nations and World Health

Organization Expert Consultation Report. Food and Agriculture

Organization of the United Nations and World Health

Organization Working Group Report.

<http://www.fao.org/es/ESN/food/foodandfood_probio_en.stm\#papers>

(Version current at May 25, 2004). 
7. Alvarez-Olmos MI, Oberhelman RA. Probiotic agents and infectious diseases: A modern perspective on a traditional therapy. Clin Infect Dis 2001;32:1567-76.

8. Mack DR, Michail S, Wei S, McDougall L, Hollingsworth MA. Probiotics inhibits enteropathogenic $E$ coli adherence in vitro by inducing intestinal mucin gene expression. Am J Physiol 1999;276:G941-50.

9. Rigothier MC, Maccanio J, Gayrol P. Inhibitory activity of Saccharomyces yeasts on the adhesion of Entamoeba histolytica trophozoites to human erythrocytes in vitro. Parasitol Res 1994;80:10-5.

10. Silva M, Jacobus NV, Deneke C, Gorbach SL. Antimicrobial substance from a human lactobacillus strain. Antimicrob Agents Chemother 1987;31:1231-3.

11. Pothoulakis C, Kelly CP, Joshi MA, et al. Saccharomyces boulardii inhibits Clostridium difficile toxin A binding and enterotoxicity in rat ileum. Gastroenterology 1993;104:1108-15.

12. Wilson KH, Perini I. Role of competition for nutrients in suppression of Clostridium difficile by the colonic microflora. Infect Immun 1988;56:2610-4.
13. Kaila M, Isolauri E, Soppi E, Virtanen E, Laine S, Arvilommi H. Enhancement of the circulating antibody secreting cell response in human diarrhea by a human lactobacillus strain. Pediatr Res 1992;32:141-4.

14. Majamaa H, Isolauri E, Saxelin M, Vesikari T. Lactic acid bacteria in the treatment of acute rotavirus gastroenteritis. J Pediatr Gastroenterol Nutr 1995;20:333-8

15. Kaila M, Isolauri E, Maija S, Arvilommi H, Vesikari T. Viable versus inactivated lactobacillus strain GG in acute rotavirus diarrhoea. Arch Dis Child 1995;72:51-3.

16. Nanno M, Ohwaki M, Mutai M. Induction by Lactobacillus casei of increase in macrophage colony-forming cells and serum colonystimulating activity in mice. Jpn J Cancer Res 1986;77:703-10.

17. D'Souza AL, Rajkumar C, Cooke J, Bulpitt CJ. Probiotics in prevention of antibiotic associated diarrhoea: Meta-analysis. BM] 2002;324:1361

18. Huff BA. Caveat emptor - "Probiotics" might not be what they seem. Can Fam Physician. 2004;50:583-7.

19. Hamilton-Miller JM, Shah S, Smith CT. "Probiotic" remedies are not what they seem. BMJ 1996;312:55-6. 


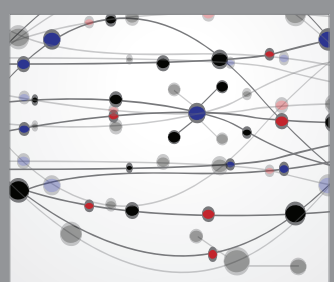

The Scientific World Journal
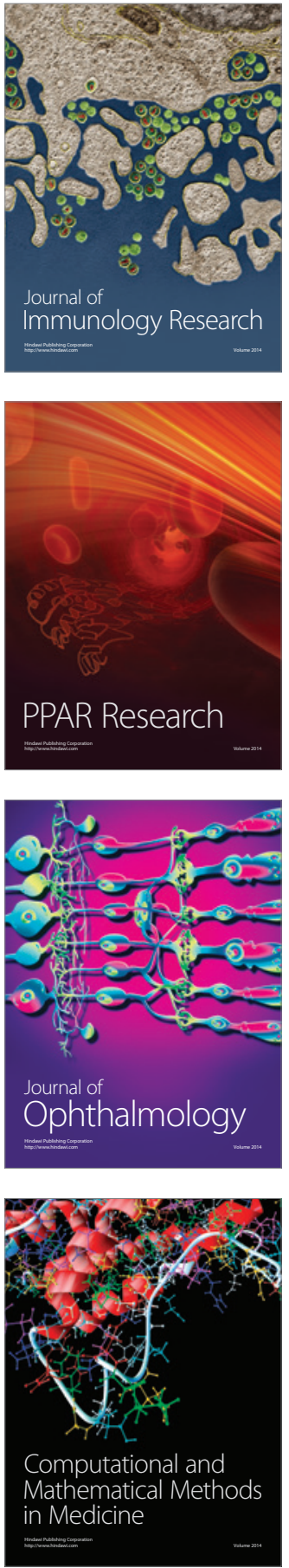

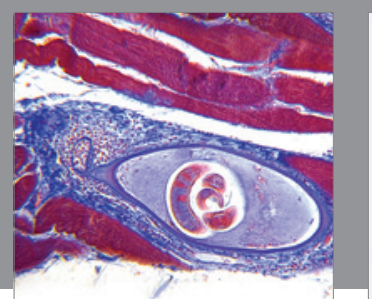

Gastroenterology Research and Practice

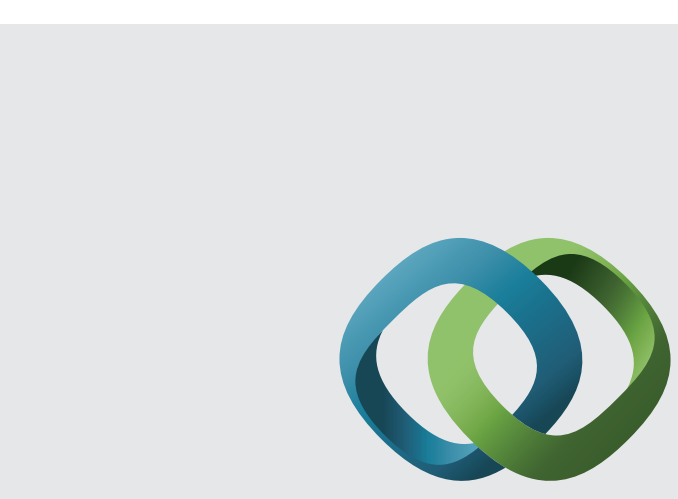

\section{Hindawi}

Submit your manuscripts at

http://www.hindawi.com
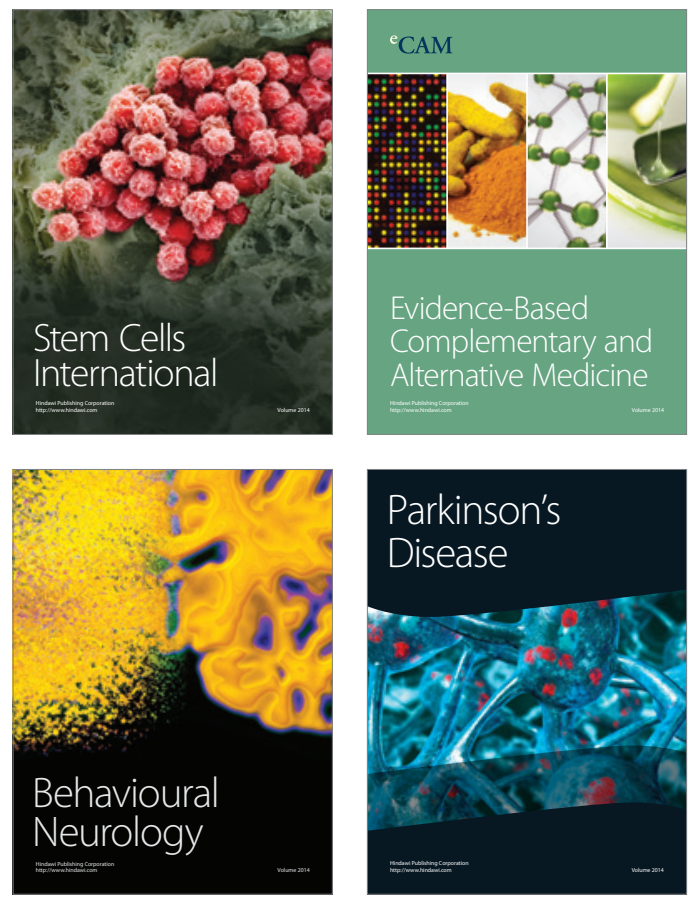
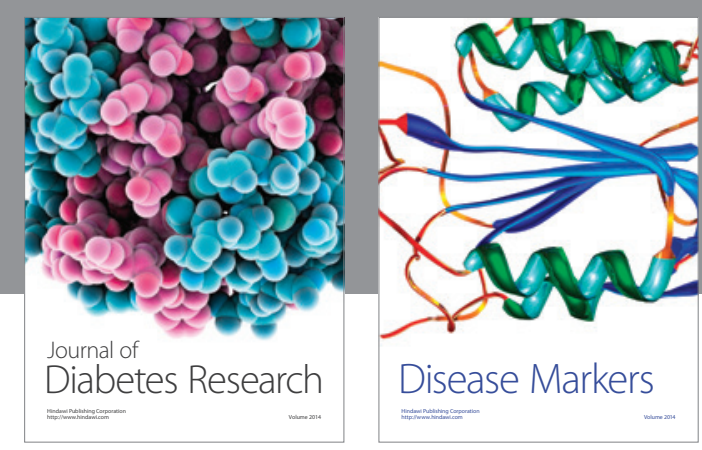

Disease Markers
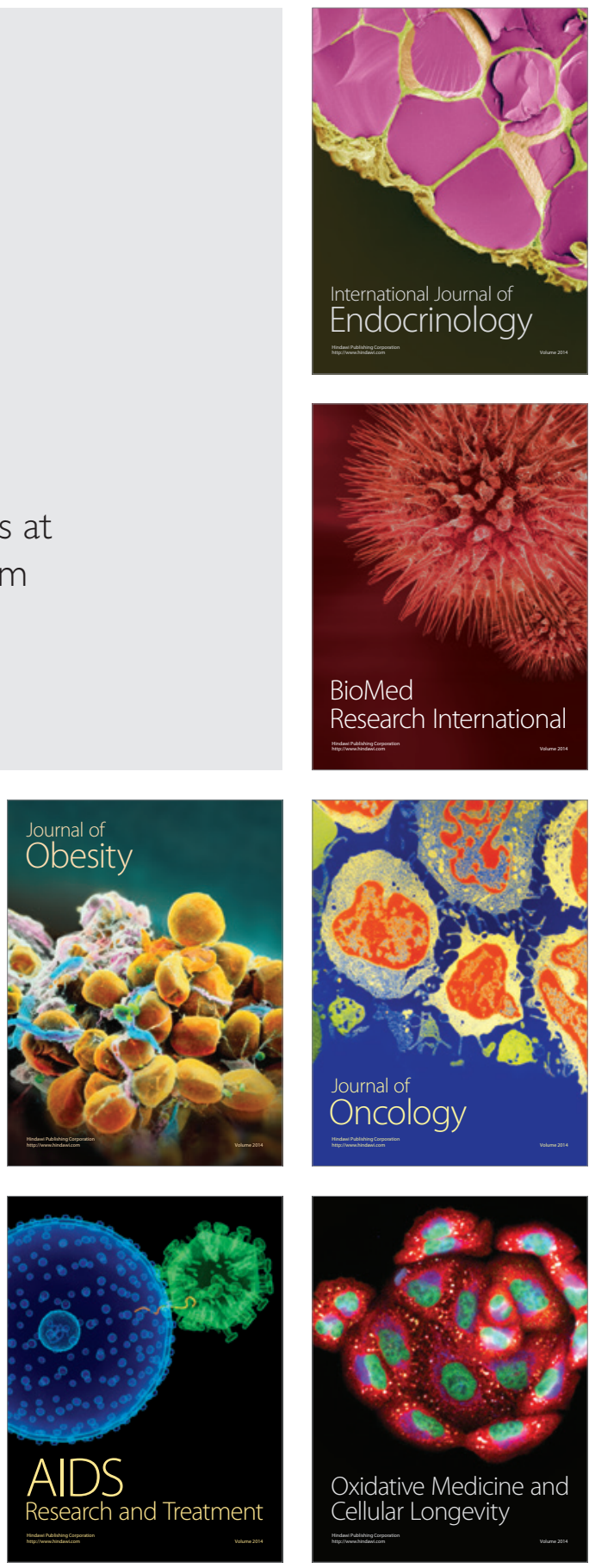